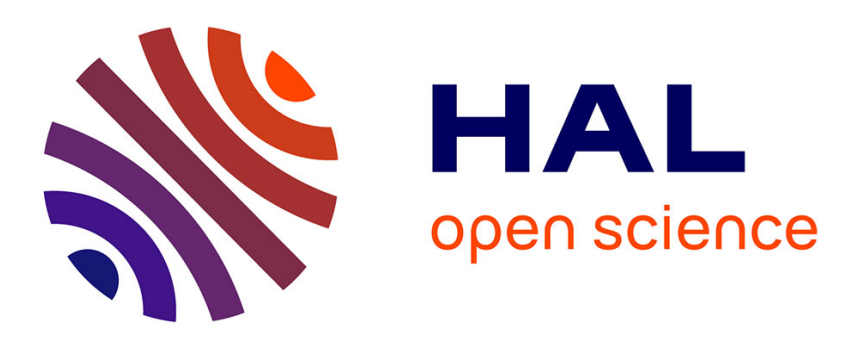

\title{
Highly efficient gene transfer into hepatocyte-like HepaRG cells: new means for drug metabolism and toxicity studies
}

Véronique Laurent, Aurore Fraix, Tristan Montier, Sandrine Cammas-Marion, Catherine Ribault, Thierry Benvegnu, Paul-Alain Jaffres, Pascal Loyer

\section{To cite this version:}

Véronique Laurent, Aurore Fraix, Tristan Montier, Sandrine Cammas-Marion, Catherine Ribault, et al.. Highly efficient gene transfer into hepatocyte-like HepaRG cells: new means for drug metabolism and toxicity studies. Biotechnology Journal, 2010, 5 (3), pp.314. 10.1002/biot.200900255 . hal00552337

\section{HAL Id: hal-00552337 \\ https://hal.science/hal-00552337}

Submitted on 6 Jan 2011

HAL is a multi-disciplinary open access archive for the deposit and dissemination of scientific research documents, whether they are published or not. The documents may come from teaching and research institutions in France or abroad, or from public or private research centers.
L'archive ouverte pluridisciplinaire HAL, est destinée au dépôt et à la diffusion de documents scientifiques de niveau recherche, publiés ou non, émanant des établissements d'enseignement et de recherche français ou étrangers, des laboratoires publics ou privés. 


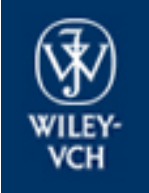

Biotechnology Journal

\section{Highly efficient gene transfer into hepatocyte-like HepaRG cells: new means for drug metabolism and toxicity studies}

\begin{tabular}{|c|c|}
\hline Journal: & Biotechnology Journal \\
\hline Manuscript ID: & biot.200900255.R1 \\
\hline Wiley - Manuscript type: & Technical Report \\
\hline $\begin{array}{r}\text { Date Submitted by the } \\
\text { Author: }\end{array}$ & 04-Jan-2010 \\
\hline Complete List of Authors: & $\begin{array}{l}\text { LAURENT, Véronique; Inserm, Unit } 522 \\
\text { FRAIX, Aurore; Université de Brest UBO, UMR6521 CNRS } \\
\text { MONTIER, tristan; Université de Brest UBO, Inserm U613 } \\
\text { CAMMAS-MARION, Sandrine; Ecole Nationale Supérieure de Chimie } \\
\text { de Rennes, UMR } 62262 \text { CNRS } \\
\text { RIBAULT, Catherine; Inserm, Unit } 522 \\
\text { BENVEGNU, Thierry; Ecole Nationale Supérieure de Chimie de } \\
\text { Rennes, UMR } 62262 \text { CNRS } \\
\text { Jaffres, Paul-Alain; Université de Brest UBO, UMR6521 CNRS } \\
\text { LOYER, Pascal; Inserm, Unit } 522\end{array}$ \\
\hline Primary Keywords: & Mammalian Biotechnology \\
\hline Secondary Keywords: & Gene expression \\
\hline Keywords: & Transfection, HepaRG cells, Electroporation \\
\hline
\end{tabular}

\section{今 scholarONE" \\ Manuscript Central}




\author{
Technical Report ((3989 words)) \\ Highly efficient gene transfer into hepatocyte-like HepaRG cells: new means for drug \\ metabolism and toxicity studies.
}

\title{
VERONIQUE LAURENT $^{1-5}$, AURORE FRAIX $^{2-5}$, TRISTAN MONTIER ${ }^{3-5}$, SANDRINE CAMMAS-MARION ${ }^{4-5}$, CATHERINE RIBAULT ${ }^{1}$, THIERRY BENVEGNU ${ }^{4-5}$, PAUL-ALAIN JAFFRES $^{2-5}$, PASCAL LOYER ${ }^{1-5}$
}

Key words : Transfection, HepaRG cells, electroporation, cationic lipids, CYP2E1

${ }^{1}$ INSERM U991; IFR140 ; Université de Rennes 1, "Foie, Métabolisme et Cancer », Hôpital Pontchaillou, Avenue de la Bataille Flandres-Dunkerque, 35033 Rennes Cedex, France.

${ }^{2}$ CEMCA, Equipe "Phosphore et vectorisation», UMR 6521 CNRS, Faculté des Sciences et Techniques, Université de Bretagne Occidentale, 6 Avenue Le Gorgeu, CS9383729838 BREST cedex 03, France.

${ }^{3}$ INSERM U613 «Génétique Moléculaire et Epidémiologie Génétique », Hôpital Morvan, CHU de Brest, Institut de Synergie des Sciences et de la Santé, 5 Avenue du Maréchal Foch, 29200 Brest, France.

${ }^{4}$ Ecole Nationale Supérieure de Chimie de Rennes (ENSCR), UMR 6226 CNRS « Sciences chimiques de Rennes», Equipe "Chimie organique et supramoléculaire», Avenue du Général Leclerc CS 50837, 35708 Rennes cedex 7, France, Université Européenne de Bretagne.

${ }^{5}$ Plateform IBiSA « SynNanoVect », Biogenouest ${ }^{\circledR}$, Brest-Rennes, France.

Corresponding author: Pascal Loyer, INSERM U522, Hôpital Pontchaillou, 35033 Rennes, France. Tel: (33).2.99.54.37.37. email: pascal.loyer@univ-rennes1.fr 


\begin{abstract}
HepaRG progenitor cells are capable to differentiate into hepatocyte-like cells expressing a large set of liver specific functions. These cells, however, express little amounts of an important cytochrome P450, the CYP2E1, which limits their use for toxicological studies of drugs metabolized by this pathway. We aimed at establishing efficient protocols of transfection in order to increase CYP2E1 expression in HepaRG cells. Transfection protocols of the GFP reporter gene were evaluated using electroporation and cationic lipids belonging to the lipophosphonates, lipophosphoramidates and lipids derived from glycine betaine. Following optimization of the charge ratios, plasmid DNA amounts and formulations with neutral co-lipids, the lipophosphoramidate compounds KLN47 and BSV10 allowed expression of the GFP in $\sim 50 \%$ of adherent progenitor HepaRG cells while electroporation targeted GFP expression in $\sim 85 \%$ of both progenitor and differentiated cells in suspension. Then, transient enforced expression of active CYP2E1 was achieved in progenitors and/or differentiated HepaRG cells using the electroporation and the lipophosphoramidate compound BSV10. Importantly, in electroporated cells, CYP2E1 expression level was correlated with a significant increase in CYP2E1specific enzymatic activity, which opens new perspectives for this CYP-dependent drug metabolism and toxicity studies using HepaRG cells.
\end{abstract}


Differentiated hepatocytes perform many complex metabolic processes including synthesis and secretion of plasma proteins, synthesis and storage of glycogen and detoxication of endogenous toxins and xenobiotics. To study the process of differentiation and to characterize the enzymatic activities in the hepatocytes two main categories of in vitro models have been developed over the past 20 years: the primary cultures of normal hepatocytes and the hepatoma cell lines. Human hepatocytes can be plated in culture conditions maintaining cell survival and expression of liver specific functions for several days to weeks [1]. Although, these models have proven to be relevant for studying some aspects of the liver biology such as induction of drug metabolism enzymes (DMEs) by xenobiotics, large variations in functional activities especially for cytochrome P450 enzymes (CYP) have been observed in human hepatocytes isolated from different donors [1]. In addition, the relative shortage and unpredictable availability of human biopsies along with the limited growth activity and lifespan of hepatocytes in primary culture limit the use of these models.

Alternatively, hepatoma cell lines have been established from differentiated hepatocellular carcinomas that retain various expression levels of liver specific proteins. These cells contain low CYP activities [2], which limits their use for studying liver metabolism and toxicity of xenobiotics. Enforced expression of CYPs has been obtained in hepatoma cells by transient or stable transfection of expression vectors encoding the CYPs themselves or liver-specific transcription factor such as C/EBP alpha [3]. Although these transfected cells are relevant models to identify DMEs involved in metabolism of new drugs, high expression levels of a limited number of transfected CYPs occur in absence of the full panel of DMEs from phases I, II and III required for the complete metabolism of xenobiotics as observed in the liver.

The human hepatoma cell line HepaRG derived from a hepatocellular carcinoma [4] circumvents these drawbacks by expressing most of the liver specific functions including high levels of the major CYPs such as CYP3A4 and CYP1A2 [5, 6]. Progenitors HepaRG cells that differentiate into hepatocyte-like cells also represent a unique in vitro cell system to investigate the process of differentiation from hepatic progenitor cells towards hepatocyte-like cells [7] as well as the phenomenon of transdifferentiation from hepatocyte to biliary phenotypes or vice versa [7]. In addition, this hepatoma cell line provides a valuable in vitro model for investigating replication of the 
Hepatitis B Virus [4], drug metabolism [5, 6, 8] as well as studies of genotoxic compounds [9]. However, the relatively low levels of the CYP2E1 enzyme in HepaRG cells $[5,6]$ limit their use for analyzing biotransformation of xenobiotics specifically metabolized by this DME. The CYP2E1 plays a major role in the metabolism of many xenobiotics in all humans [10]. Thus, expression of higher levels of CYP2E1, achieved by transfection in differentiated HepaRG cells, would be particularly useful to complete the pathways of biotransformation in these cells.

It is generally accepted that hepatic cells, especially primary hepatocytes, are difficult to transfect [11]. Liposome mediated transient transfection using the most recent cationic lipids allowed gene delivery in 10 to $30 \%$ of rodent and human hepatocytes in primary culture. These protocols were useful to study regulatory gene sequences [11] but remained of limited help to study the effect(s) of enforced expression of gene of interest in whole cell populations because of the relatively low rate of transfection.

Because of the raising interest in HepaRG cells for drug metabolism studies and for analyzing the process of differentiation from hepatic progenitors towards hepatocytes, optimization of HepaRG cell transfection has become a pertinent task. The aim of this work was first to establish efficient protocols of transfection of both progenitor and differentiated HepaRG cells, then to achieve enforced expression of CYP2E1 protein.

The HepaRG cells are available from Dr. Guillouzo (christiane.guillouzo@univ-rennes1.fr) for academic laboratories and from Dr. Chesné (christophe.chesne@biopredic.com) for industrial laboratories. Progenitor HepaRG cells were cultured as previously described $[4,5,7]$. For full expression of liver specific genes in hepatocyte-like cells, the culture medium was supplemented with $2 \%$ DMSO for 2 more weeks [5].

A panel of commercial transfection reagents (Supplemental data S1) and the pEGFP-C3 (Clontech, Saint Germain en Laye, France) expression vector encoding the Green Fluorescent Protein (GFP) were used to transfect HepaRG cells. GFP positive cells were detected $48 \mathrm{~h}$ after transfection using the FC500 cytometer (Beckman Coulter, France). The mean of fluorescence (Geo mean) was determined for the GFP positive cell population. To determine background fluorescence, HepaRG cells were transfected with the non coding pC3 plasmid (pEGFP-C3 lacking the GFP cDNA). Cell 
viability was assessed using the Side Scatter (SSC) versus Forward Scatter (FSC) dot plot. Previous experiments showed a clear decrease in size (FSC) and increase in structure (SSC) in HepaRG cells undergoing necrosis or apoptosis compared to viable cells. GFP positive cells were visualized with a Zeiss fluorescent microscope and the AxioVision LE Rel. 4.3 software.

In progenitor HepaRG cells, Lipofectamine $2000^{\circledR}$ was the most potent transfection reagent with an efficiency reaching $25 \%$ of GFP positive cells but with a strong toxicity. Other reagents allowed GFP expression in only 10 to $15 \%$ of cells. In differentiated hepatocyte-like cells, transfection rates were even lower (1 to $14 \%$ ) than in progenitor cells (Supplemental data S1).

We next evaluated the lipophosphonates GLB43 and EG316 and lipophosphoramidates KLN25, KLN47 and BSV10 (Figure 1) [12, 13]. We also evaluated the MM18 compound belonging to a family of glycine betaine lipids efficient for transfection in mouse hepatocytes [14]. The lipophosphonates GLB43 and EG316, lipophosphoramidates KLN47 and BSV10 have been previously reported [12]. For the KLN25 and the glycine betaine MM18 [13], the synthesis are detailed in Supplemental data S2 and S3. All lipids are presented in Supplemental data S4 and the preparation of liposomes is described in Supplemental data S5. Transfections with the lipophosphonates, lipophosphoramidates and the MM18 produced by the IBiSA plateform "SynNanoVect" from Biogenouest ${ }^{\circledR}$ (France) were performed using $10^{5}$ cells/well (in 24-multiwells) plated $24 \mathrm{~h}$ prior transfection. Liposome suspensions (10 $\mathrm{mg}$ of lipids $/ \mathrm{mL}$ ) were sonicated using an ultrasound water bath sonicator for 5 minutes. Liposomes and plasmids $(0.5 \mu \mathrm{g})$ were diluted in $100 \mu \mathrm{L}$ OptiMEM medium (Gibco) prior mixing DNA plasmid dilution with liposome suspension. The resulting lipoplex solutions were vortexed for 30 seconds, incubated at room temperature for 30 minutes, added dropwise to the culture medium of HepaRG cells deprived in antibiotics and incubated for 4 hours with cells prior culture medium renewal.

A broad range of charge ratios from 0.5 to 16 was tested with $0.8 \mu \mathrm{g}$ of DNA plasmid (Figure 1). Different liposomal compositions were also studied including either the cationic lipid alone or associated with the neutral co-lipids DOPE or cholesterol, known to promote fusion between the lipoplexe and cellular membranes and to increase lipoplexe stability, respectively. The KLN47 was 
used alone since we previously showed that its association with DOPE and cholesterol strongly reduced plasmid DNA delivery.

For the EG316, GLB43 and KLN25 liposomes (Figure 1A, B and C), the highest transfection efficiencies in progenitor HepaRG cells were $16 \pm 3,14 \pm 1$ and $7 \pm 1 \%$, respectively, at the optimal charge ratio of 2. The cell viability decreased with high charge ratio. With BSV10 lipid (Figure 1D), the percentage of GFP positive cells increased proportionally to the charge ratio up to $\sim 20 \%$ at the ratios 8 and 16 but was associated with low cell viabilities. MM18 lipid was the less efficient compound ( $5 \%$ of GFP cells) with reduced cell viability at high charge ratios (Figure 1E). The KLN47 compound allowed the highest gene transfer in HepaRG cells with efficiencies up to $45 \%$ for the charge ratio of 4 with cell viability down to $\sim 60 \%$ of control culture (Figure $1 \mathrm{~F}$ ).

Combining lipids EG316, GLB43, KLN25, BSV10 to the co-lipid DOPE significantly improved transfection efficiencies of HepaRG cells (i.e. 40\% of GFP positive cells with BSV10DOPE, $\sim 80 \%$ cell viability at the charge ratio of 1 ). MM18 liposome formulation with $15 \%$ cholesterol also significantly increased the transfection rate up to $20 \%$ of GFP positive cells at the charge ratio of 2 (Figure 1E). The benefit of using the co-lipid DOPE is probably due to its ability to induce an intracellular transition of the liposome/DNA complex from a lamellar to a non-lamellar phase, which enhances DNA release. Similar experiments were also performed with differentiated HepaRG cells but none of these formulations gave transfection efficiencies higher than $10 \%$ (data not shown) and, thus, did not improve transfection rates obtained with commercially available reagents. In conclusion, the KLN47 and BSV10/DOPE formulations allowed high gene delivery in proliferating progenitors cells $(\sim 50 \%)$ but not in quiescent differentiated HepaRG cells in agreement with the fact that gene transfer by liposomes depend upon cell cycle [15].

Then, we evaluated the Microporator MP100 from LabTech France (Paris) designed to deliver DNA into cells in suspension [note: the device originally engineered by Digital Bio (NanoEnTek Inc., Korea) is now commercialized as the $\mathrm{Neon}^{\mathrm{TM}}$ electroporator from Invitrogen (Carlsbad, USA)]. Progenitor HepaRG cells were detached by trysinization while differentiated HepaRG cells were selectively detached by mild trypsination at room temperature [7]. Cells were washed twice with PBS 
and resuspended in buffer $\mathrm{R}\left(10^{7}\right.$ cells $\left./ \mathrm{mL}\right)$. For both progenitor and hepatocyte-like HepaRG cells, 24 conditions of electroporation were tested (Supplemental data S6).

In progenitor cells, 16 conditions gave transfection rates between 80 and $95 \%$ of GFP positive cells with cell viabilities ranging from 65 to $90 \%$ (Figure 2A). Other settings led to lower transfection efficiencies, which, however, remained above $50 \%$ of GFP positive cells with high cell viability rates. In hepatocyte-like cells, electroporation also allowed efficient DNA delivery and high expression levels of the GFP proteins since two conditions (C5 and 4) reached transfection and cell viability rates above $80 \%$ (Figure 2B). The other settings led to transfection efficiencies ranging from 25 to $78 \%$ with cell viabilities varying considerably.

These data demonstrated that in HepaRG cells, electroporation is a more efficient technology for gene delivery than the cationic lipids tested. However, the transfection using the lipophosphoramidate compounds KLN47 and BSV10 will be useful since gene delivery is performed with adherent progenitor cells in contrast with the electroporation of cell suspensions. The high rate of transfection in differentiated HepaRG cells by electroporation confirms the notion that electroporation facilitates the nuclear translocation of plasmid DNA in poorly proliferating cells [16].

We next addressed the question of the relationship between the efficiency of transfection and the amount of plasmid DNA. The percentage of GFP positive cells and the mean of fluorescence were measured according to the amounts of DNA ranging (from $10 \mathrm{ng}$ to $2 \mu \mathrm{g} / 10^{5}$ cells) using electroporation and the liposomes BSV10/DOPE, GLB43/DOPE and MM18/Chol (Supplemental data S7).

For electroporation, the smallest amount of DNA (10 ng) led to 20\% of GFP positive cells. This percentage was linear up to $0.2 \mu \mathrm{g}$ of plasmid to reach $\sim 70 \%$ of GFP expressing cells. For 0.3 to $0.6 \mu \mathrm{g}$ of plasmid, the maximal efficiency was reached $(\sim 85 \%)$ while high amounts up to $2 \mu \mathrm{g}$ of plasmid slightly decreased the rate of transfection. The mean of fluorescence did not correlate the percentage of GFP positive cells for the small amounts of DNA but increased abruptly to reach the highest fluorescence intensity for the amounts between 0.3 and $0.6 \mu \mathrm{g}$ of plasmid. 
For BSV10/DOPE, GLB43/DOPE and MM18/Chol liposomes, optimal transfection efficiencies were obtained for $0.4,1$ and $0.8 \mu \mathrm{g}$ of plasmid, respectively, and the mean of fluorescence correlated well with the percentage of GFP positive cells. GFP expression remained very low $(<5 \%)$ up to $0.1 \mu \mathrm{g}$ of plasmid and was not proportional to the amount of DNA while between 0.1 and $0.4 \mu \mathrm{g}$ \pm 0.2 of DNA, this percentage was linear.

Then, we studied whether the percentages of GFP positive cells were correlated to the intracellular amount of plasmid. Percentages of GFP progenitor HepaRG cells, transfected with the pEGFPc3, were compared to the ratio of fluorescence between cells transfected with the non coding rhodamine-labelled and unlabeled pC3 plasmids (Supplemental data S8). Transfection by electroporation or with the BSV10/DOPE liposomes that allowed 80 and $50 \%$ of GFP positive cells were characterized by small fluorescence ratios of 3.5 and 4.5 , respectively. In contrast, the GLB43/DOPE and MM18/Chol liposomes, which triggered only 15 and 5\% of GFP expression, exhibited high fluorescence ratios of 8 and 12. In situ visualization of FITC-labelled pC3 plasmid confirmed that cells transfected with MM18/Chol liposomes exhibited larger and more intense fluorescent dots than electroporated cells. These data further confirm that the improvement of nucleocytoplasmic transport of plasmid DNA following non viral gene transfer is a key parameter to achieve high gene expression [17].

Following the optimization of the transfection in HepaRG cells, we next assayed the transient ectopic expression of the CYP2E1 (Figure 3). The human CYP2E1 cDNA (obtained from Dr. De Waziers, INSERM U775, Université Paris Descartes, France) was amplified by PCR using the primers ATGTCTGCCCTCGGAGTCACC (foward) and TCATGAGCGGGGAATGACACA (reverse) and inserted into the pcDNA3.1/V5-His-Topo. Both progenitor and differentiated hepatocyte-like HepaRG cells were transfected with the pEGFPc3 or the human CYP2E1 encoding vector using electroporation (Figure 3A) and the lipids BSV10 and KLN47 (Figure 3C). For immunoblotting of the GFP (antibody: T-19, sc5384, Santa Cruz Biotechnology), the CYP3A4 (antibody: AB1254, Chemicon), the CYP2E1 (antibody: Oxford Biomedical, USA) and the HSC70 (antibody: B-6, sc7298, Santa Cruz Biotechnology), cells were lysed in $50 \mathrm{mM}$ Hepes pH 7.9, $150 \mathrm{mM} \mathrm{NaCl}, 0.1 \mathrm{mM}$ EDTA, 10\% glycerol, $0.5 \%$ Tween 20 supplemented with protease inhibitors (EDTA-free, Roche). Ten $\mu \mathrm{g}$ of total 
proteins were resolved on $12.5 \%$ SDS-PAGE (NewPage, Invitrogen), transferred onto nitrocellulose membrane and immunoblotted.

As expected, we evidenced that CYP2E1 and CYP3A4 were undetectable in progenitor cells while hepatocyte-like cells expressed high levels of CYP3A4 and low amounts of CYP2E1 (Figure 3A). In contrast, progenitors and differentiated HepaRG cells electroporated with the CYP2E1 vector expressed much higher levels of this DME while the CYP3A4 level in hepatocyte-like cells was not affected by electroporation (Figure 3A). Interestingly, the highest levels of GFP and CYP2E1 were observed in hepatocyte-like HepaRG cells although the percentages of GFP positive cells are very similar in both progenitor and differentiated cells (Figure 2). This is most likely due to the high overall protein synthesis in differentiated versus progenitor cells. Importantly, the CYP2E1 expression level in transfected cells was correlated with a $\sim 5$ to 6 fold increase in the CYP2E1-specific enzymatic activity compared to the activity in the control cells (Figure 3B).

We also evidenced the expression of the GFP and CYP2E1 proteins in the progenitor cells transfected with the BSV10/DOPE formulation and in a much lesser extend with the lipid KLN47 (Figure 3C). However, in differentiated cells, we did not observe any significant increase in CYP2E1 expression following transfection with these lipids while the GFP was detected at low levels (Figure $3 C)$.

Together, these data demonstrated that using the electroporation, we achieved the enforced expression of the human CYP2E1 in differentiated HepaRG cells. This drug-metabolizing enzyme is of an enormous toxicological and carcinogenic importance because of its implication in the metabolism of a large number of chemicals such as ethanol, acetaminophen, acrylamide and many cancer suspected agents [10]. Thus, low expression of CYP2E1 in differentiated HepaRG cells was a clear limitation in their use for metabolism and toxicological studies of a wide range of molecules with relevance for human health. Differentiated HepaRG cells expressing high levels of the most important CYPs including the CYP2E1 definitely opens new perspectives for drug metabolism and toxicity studies of a variety of new drugs. 


\section{ACKNOWLEGEMENT}

We thank D. Glaise for technical assistance with HepaRG cell culture, Dr. C. Chesné and G. Ribault (Biopredic Int.) for measurement of the CYP2E1 activity, Dr. L. Lemiègre for helping with the synthesis of the MM18 glycine betaine lipid and Dr F. Desmots for helping with the design of the figures. Flow cytometry was performed with the core facility of the Institut Fédératif de Recherche de Rennes (IFR140). V. L. was a recipient of fellowships from «l'association de transfusion sanguine et de biogénétique Gaëtan Saleün » (EFS, Brest) and «la Ligue Contre le Cancer». A. F. is a recipient of a fellowship from «Ministère de la Recherche et de la Technologie». This work was funded by INSERM, Institut Fédératif de Recherche de Rennes (IFR140), Région Bretagne (PRIR VectSynth), the GIS-IBiSA and the European Commission FP7 program "LIV-ES" (HEALTH-F5-2008-223317).

The authors have declared no conflict of interest. 


\section{REFERENCES}

[1] Guillouzo, A., Guguen-Guillouzo, C., Evolving concepts in liver tissue modeling and implications for in vitro toxicology. Expert Opin Drug Metab Toxicol, 2008, 4,1279-1294.

[2] Wilkening, S., Stahl, F., Bader A., Comparison of primary human hepatocytes and hepatoma cell line HepG2 with regard to their biotransformation properties. Drug Metab. Dispos., 2003, 31, 1035-1042.

[3] Jover, R., Bort, R., Gomez-Lechon, M.J., Castell, J.V., Re-expression of C/EBP alpha induces CYP2B6, CYP2C9 and CYP2D6 genes in HepG2 cells. FEBS Lett., 1998, 431, 227-230.

[4] Gripon, P., Rumin, S., Urban, S., Le Seyec, J. et al, Infection of a human hepatoma cell line by hepatitis B virus. Proc Natl Acad Sci USA., 2002, 99, 15655-60.

[5] Aninat, C., Piton, A., Glaise, D., Le Charpentier, T. et al., Expression of cytochromes P450, conjugating enzymes and nuclear receptors in human hepatoma HepaRG cells. Drug Metab Dispos. 2006, 34, 75-83.

[6] Kanebratt, K.P., Andersson, T.B., Evaluation of HepaRG cells as an in vitro model for human drug metabolism studies. Drug Metab Dispos., 2008, 36, 1444-52.

[7] Cerec, V., Glaise, D., Garnier, D., Morosan, S. et al., Transdifferentiation of hepatocyte-like cells from the human hepatoma HepaRG cell line through bipotent progenitor. Hepatology, 2007, 45, 957-67. 
[8] Guillouzo, A., Corlu, A., Aninat, C., Glaise, D. et al., The human hepatoma HepaRG cells: a highly differentiated model for studies of liver metabolism and toxicity of xenobiotics. Chem Biol Interact., 2007, 168, 66-73.

[9] Jossé, R., Aninat, C., Glaise, D., Dumont, J. et al., Long-term functional stability of human HepaRG hepatocytes and use for chronic toxicity and genotoxicity studies. Drug Metab. Dispos., 2008, 36, 1111-1118.

[10] Gonzales, F.J., The 2006 Bernard B. Brodie Award lecture : CYP2E1. Drug Metab. Dispos., $2007,35,1-8$.

[11] Ourlin, J.C., Vilarem, M.J., Daujat, M., Harricane, M.C. et al., Lipid-mediated transfection of normal adult human hepatocytes in primary culture. Anal Biochem., 1997, 247, 34-44.

[12] Mével, M., Breuzard, G., Yaouanc, J.-J., Clément, J.-C. et al., Synthesis and transfection activity of new cationic phosphoramidate lipids: high efficiency of an imidazolium derivative. Chem.Bio.Chem., 2008, 9, 1462-1471.

[13] Montier, T., Benvegnu, T., Jaffrès, P.-A., Yaouanc, J.-J. et al., Progress in cationic lipid-mediated gene transfection: a series of bio-inspired lipids as an example. Curr. Gene Ther., 2008, 8, 296312.

[14] Gilot, D., Miramon, M.L., Benvegnu, T., Ferrieres, V. et al., Cationic lipids derived from glycine betaine promote efficient and non-toxic gene transfection in cultured hepatocytes. J Gene Med., 2002, 4, 415-27. 
[15] Brunner, S., Sauer, T., Carotta, S., Cotten, M., Saltik, M., Wagner, E., Cell cycle dependence of gene transfer by lipoplex, polyplex and recombinant adenovirus. Gene Ther., 2000, 7, 401-407.

[16] Brunner, S., Fürtbauer, E., Sauer, T., Kursa, M. et al., Overcoming the nuclear barrier : cell cycle independent nonviral gene transfer with linear polyethylenimine or electroporation. Mol. Ther., 2002, 5, 80-86.

[17] Lechardeur, D., Lukacs, G.L., Nucleocytoplasmic transport of plasmid DNA: a perilous journey from the cytoplasm to the nucleus. Hum. Gene Ther., 2006, 17, 882-889. 


\section{FIGURE LEGENDS}

Figure 1 : Transfection of HepaRG cells with the cationic lipophosphonates, lipophosphoramidates and glycine betaine lipids.

The lipophosphonates EG316 (A) and GLB43 (B) and lipophosphoramidate KLN25 (C), BSV10 (D) and KLN47 (F) and the glycine betaine lipid MM18 (E) were used to transfect progenitor HepaRG cells. $10^{5}$ adherent cells were transfected with $0.8 \mu \mathrm{g}$ of pEGFPC 3 vector and cationic lipids either used alone (charts of the left side) or combined with neutral the co-lipid DOPE or cholesterol (Chol) [charts of the right side] at different charge ratios (from 0.5 to 16: abscissa axis). GFP expression (histograms) and cell viability (lines) were detected by flow cytometry $48 \mathrm{~h}$ after transfection. Background fluorescence was determined for all liposome formulations using the non coding pc3 DNA plasmid. Results of three independent experiments are expressed as percentages of GFP positive cells and cell viability compared to non transfected control cells (NT).

Figure 2 : Transfection of progenitor and differentiated HepaRG cells by electroporation.

The Microporator MP100 [Neon ${ }^{\mathrm{TM}}$ system from Invitrogen (Carlsbad, USA)] designed to deliver DNA into cells in suspension was used to transfect $0.5 \mu \mathrm{g}$ of pEGFPC3 expression vector encoding the GFP reporter gene in $10^{5}$ progenitor (A) and differentiated (B) HepaRG cells. GFP expression (histograms) and cell viability (lines) were detected by flow cytometry. Results of three independent experiments are expressed as percentages of GFP positive cells and cell viability compared to non transfected control cells (NT). Progenitor and differentiated HepaRG cells expressing GFP were visualized by fluorescence microscopy and phase contrast (photographs on the right side, white bar: 20 $\mu \mathrm{m})$. 
Figure 3 : Transient enforced expression of the CYP2E1 in differentiated HepaRG cells

Progenitor (Prog. cells) and differentiated HepaRG (Diff. cells) cells were electroporated (A) or transfected with the BSV10/DOPE and KLN47 liposomes (C) and the GFP or CYP2E1 expression vector (+ indicates the vector used). The expression levels of the CYP3A4, CYP2E1 and the GFP were analyzed by immunoblotting and Hsc70 was used as loading control. First lane of panel C: differentiated HepaRG electroporated (EP) with the CYP2E1 vector. In electroporated cells, the CYP2E1-specific enzymatic activities were measured using the chlorzoxazone as substrate followed by detection of the 6-hydroxychlorzoxazone metabolite by HPLC (B). Results of three independent experiments are expressed as the fold increase in CYP2E1-specific enzymatic activity compared to the basal activity (arbitrarily set as 1) in differentiated HepaRG cells transfected with the GFP vector (B). 

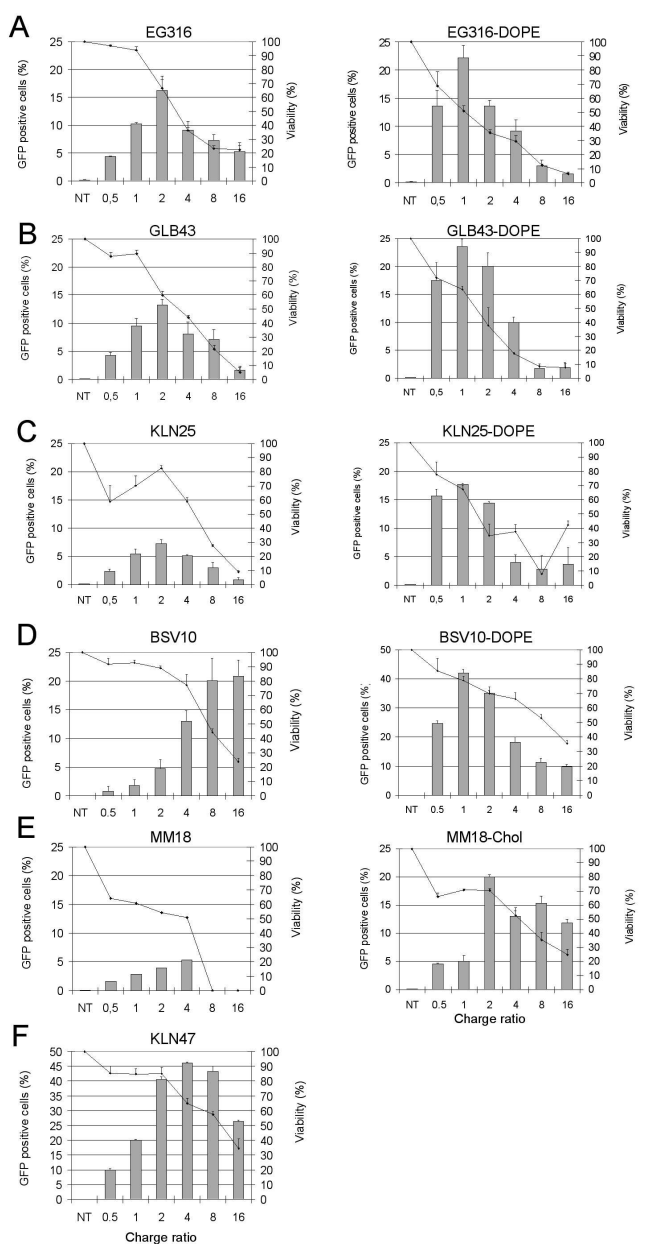

Figure 1

209×297mm (300 x 300 DPI)

50

51

52

53

54

55

56

57

58

59

60 


1
2
3
4
5
6
7
8
9
10
11
12
13
14
15
16
17
18
19
20
21
22
23
24
25
26
27
28
29
30
31
32
33
34
35
36
37
38
39
40
41
42
43
44
45
46
47
48
49
50
51
52
53
54
55
56
57
59
60
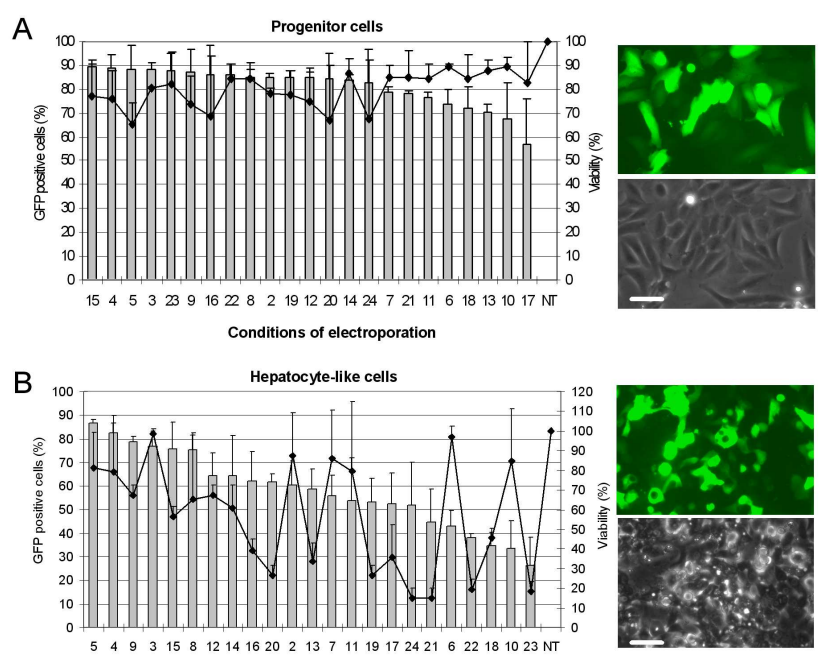

Conditions of electroporation

Figure 2

$209 \times 297 m m(300 \times 300$ DPI $)$ 


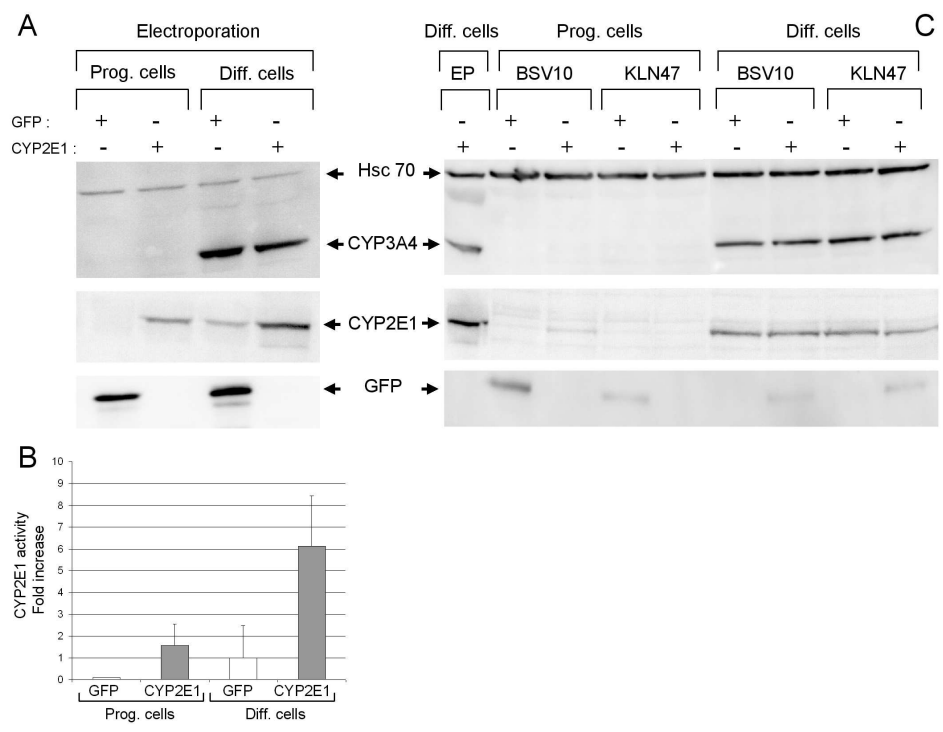

Figure 3 